\title{
Sputtering yield measurements during low energy xenon plasma bombardment
}

\author{
R. P. Doerner ${ }^{\mathrm{a})}$ \\ Center for Energy Research University of California at San Diego, La Jolla, California 92093 \\ D. G. Whyte \\ University of Wisconsin-Madison, Madison, Wisconsin 53706 \\ D. M. Goebel \\ Boeing Space and Communications, Torrance, California 90505
}

(Received 2 December 2002; accepted 18 February 2003)

\begin{abstract}
The sputtering yields of molybdenum, titanium, beryllium, and carbon have been measured during xenon ion bombardment from a plasma in the energy range between 10 and $200 \mathrm{eV}$. The erosion rates of $\mathrm{Mo}, \mathrm{Be}$, and $\mathrm{C}$ are measured both spectroscopically in the plasma and using the standard weight loss technique. Spectroscopic measurements of Ti sputtering yields, where no atomic physics data is available, are normalized to the weight loss measurements. The erosion rates of the metals decrease with the reduced mass of the metal-xenon combination and decrease with the increasing metal's binding energy, as expected. The erosion results for bombardment of graphite indicate that the sputtering rate of carbon, as atoms, from the surface is insufficient to explain the total carbon weight loss measured. The multiple mechanisms for carbon erosion during plasma bombardment are discussed and the sputter rates of carbon atoms and carbon dimers are presented. (C) 2003 American Institute of Physics. [DOI: 10.1063/1.1566474]
\end{abstract}

\section{INTRODUCTION}

The erosion rate of molybdenum due to xenon ion bombardment is a critical issue for the erosion rate of the grids in ion thruster engines. ${ }^{1}$ Detailed measurements of the erosion rate of various materials due to xenon ion bombardment have been reported previously, ${ }^{2-4}$ and the results in the literature surveyed in Ref. 5. The previous measurements have been restricted to energies above $100 \mathrm{eV}$, and at $100 \mathrm{eV}$ the literature shows a range in molybdenum sputtering yields of over 1 order of magnitude. Due to the exponential behavior of low energy sputtering, extrapolating those data to lower energies of interest for xenon ion thrusters results in large uncertainties ${ }^{6}$ that make predictions of the life of thruster grids highly suspect. The experiments reported here are the first measurements of the sputtering yields of $\mathrm{Mo}, \mathrm{Ti}, \mathrm{Be}$, and $\mathrm{C}$ by $\mathrm{Xe}$ ion bombardment in the energy range of a few hundred volts down to several tens of electron volts.

The sputtering yield measurements reported here involve the use of a xenon plasma to provide a large xenon ion flux, typically $1 \times 10^{18}$ ions $/ \mathrm{cm}^{2} \mathrm{~s}$, to samples immersed within the plasma column. The samples are biased relative to the plasma to produce ion bombardment energies from 10 to 200 $\mathrm{eV}$. The plasma is created using a reflex arc discharge in the PISCES linear plasma source ${ }^{7,8}$ at the University of California, San Diego. One of the unique features of the PISCES facility is its location within an airtight enclosure ${ }^{9}$ that allows toxic materials (such as beryllium) to be investigated. The device itself consists of a plasma source region, a main

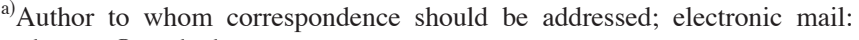
rdoerner@ucsd.edu chamber where plasma flows to a biased, temperaturecontrolled target, and a sample-loading chamber. An in situ surface analysis station is also connected to the machine and is used to determine the eroded surface composition and morphology.

Sputtering yields of the samples inserted into the plasma are determined by two techniques. The standard method is to bombard the biased sample with a constant ion flux, and measure the sample weight loss to determine the yield. This is normally done in a relatively low density (about $1 \times 10^{12}$ $\mathrm{cm}^{-3}$ ) plasma in which very few of the sputtered atoms are ionized and returned to the sample. However, care must be taken to account for any redeposition of sputtered material back onto the sample surface by the plasma. An alternative measurement method uses an absolutely calibrated optical spectrometer to measure the optical line emission of sputtered sample material that must pass through the plasma column. In this case, the plasma density is increased (up to 1 $\times 10^{13} \mathrm{~cm}^{-3}$ ) to ensure that all the sputtered atoms excite and are ionized in the viewing volume of the spectrometer. Since redeposited materials erode at the nearly same rate as the original surface, this technique is insensitive to any material returned to the surface by the plasma that complicates the weight loss measurements.

Sputtering yield results are shown for xenon ion bombardment of $\mathrm{Mo}, \mathrm{Ti}, \mathrm{Be}$, and carbon in the energy range of $10-200 \mathrm{eV}$. The molybdenum erosion yield at energies in the $100-200 \mathrm{eV}$ is found to agree well with the results published in Refs. 3 and 5. The erosion rates of the other metals investigated here are found to scale with the reduced mass of the metal- xenon combination and decrease with the increasing metal's binding energy. For example, at $100 \mathrm{eV}$, titanium is 

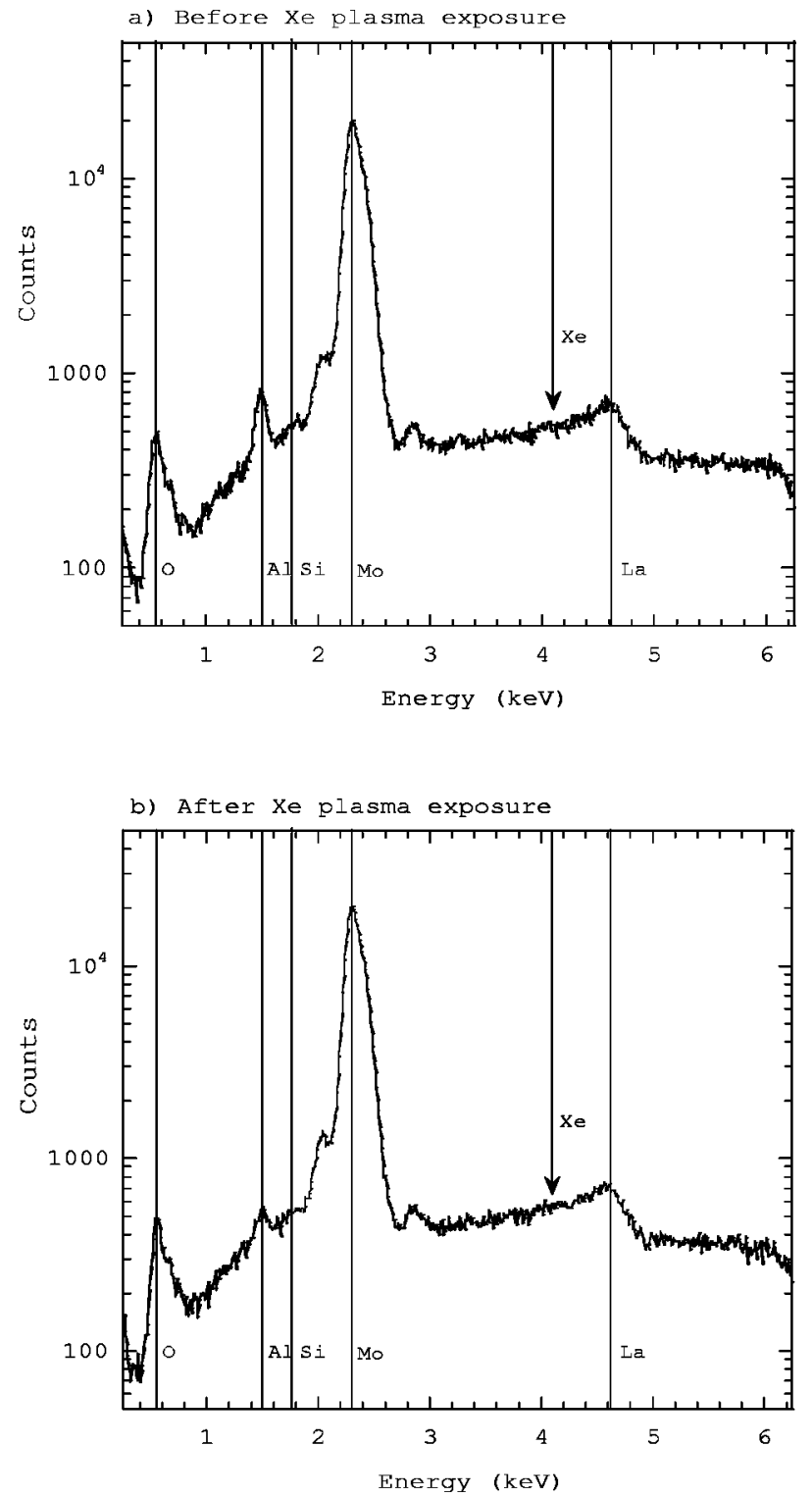

FIG. 1. EDS spectra from molybdenum samples before (a) and after (b) xenon plasma bombardment show no impurity deposition on, or xenon accumulation in, the sample.

found to have a lower yield than molybdenum by a factor of about 1.9, and beryllium is found to be a factor of about 2.6 times lower than molybdenum. Likewise, weight loss measurements of the sputtering of carbon indicate that carbon erodes at a rate about 8 times slower than molybdenum at $100 \mathrm{eV}$. Spectroscopic results indicate that the sputtering rate of carbon atoms from the Poco graphite samples is insufficient to explain the total carbon weight loss data. A discussion of the other erosion mechanisms that are likely responsible for the measured carbon sputtering yields is included.

\section{PISCES DESCRIPTION}

The PISCES-B device is a steady-state, linear plasma device. ${ }^{7,8} \mathrm{~A} 6 \mathrm{~cm}$ diam magnetized plasma column is formed along the axis of the vacuum chamber. The plasma is produced by a large area reflex discharge from a $\mathrm{LaB}_{6}$ disk cathode to a cylindrical anode. The main plasma vacuum chamber is surrounded by three electromagnetic coils that generate an axial magnetic field along the axis with typical field strengths of $0.2-1 \mathrm{kG}$. Two turbomolecular pumps with a total pumping speed of $6500 \mathrm{l} / \mathrm{s}$ are attached to the vacuum chamber to provide a base pressure in the $10^{-8}$ Torr range.

A reciprocating double-Langmuir probe is used to measure the radial profile of both the plasma temperature and density in the plasma column. The ion flux measured by the probe is verified by monitoring the current collected on the target when the target is biased to draw ion saturation current. The total uncertainty in the ion flux measurement with position is approximately $20 \%$. The facility produces steady plasmas permitting hours of continuous exposure of samples inserted into the plasma. The plasma parameters can be varied over a large range of electron temperatures $T_{e}$ (typically from 3 to $35 \mathrm{eV}$ ) and electron density $n_{e}$ (typically from $10^{11}$ to $10^{13} \mathrm{~cm}^{-3}$ ) in the target region. The plasma parameters at the target are adjusted by varying the gas flow rate and the input power level to the plasma producing discharge.

An actively cooled sample manipulator and sample holder, immersed in one end of the plasma column, permits control of the sample temperature. During these experiments the target temperatures were in the range between 100 and $200{ }^{\circ} \mathrm{C}$. The sample radius $r_{s}$ during these experiments is 1.1 $\mathrm{cm}$. Applying a negative bias to the sample manipulator and sample holder controls the bombarding energy of the ions. The distribution of the ion energy is on the order of one half the electron temperature, ${ }^{10}$ in this case plus or minus about 2 $\mathrm{eV}$. The sample manipulator cap used to clamp the sample to the temperature-controlled surface is made of the same material as the sample to avoid any potential materials contamination of the surfaces. The geometry of the experiment is such that the ions impinge normal to the sample surface.

A microprobe gas analyzer, MPA-565C from Ferran Scientific, is used to monitor the vacuum conditions before and during plasma exposure. Unlike most RGAs, this gas analyzer has a maximum measurable pressure of $10^{-2}$ Torr and is able to continuously monitor the vacuum conditions throughout the experimental run. The primary plasma impurity during these experiments was oxygen (from residual water vapor), at a level of approximately $0.1 \%$. Details of the vacuum condition have been reported in early publications. ${ }^{11,12}$

Surface analyses of the samples are performed both before and after plasma experiments using a JEOL T330A scanning electron microscope (SEM) and a KEVEX energy dispersive $\mathrm{x}$-ray detector. In situ Auger emission spectroscopy/x-ray photoelectron spectroscopy (AES/XPS) analysis is also used to monitor any change in the surface composition of the plasma-exposed sample. For the metal samples used, no change in the surface composition is detectable after plasma bombardment. Typical measurements from a Mo sample both before and after plasma exposure are shown in Figs. 1(a) and 1(b), respectively. However, after exposure of carbon samples to xenon plasma bombardment, a relatively large amount of xenon atoms (14\%) are trapped in the near-surface region of the carbon sample, Fig. 2. 


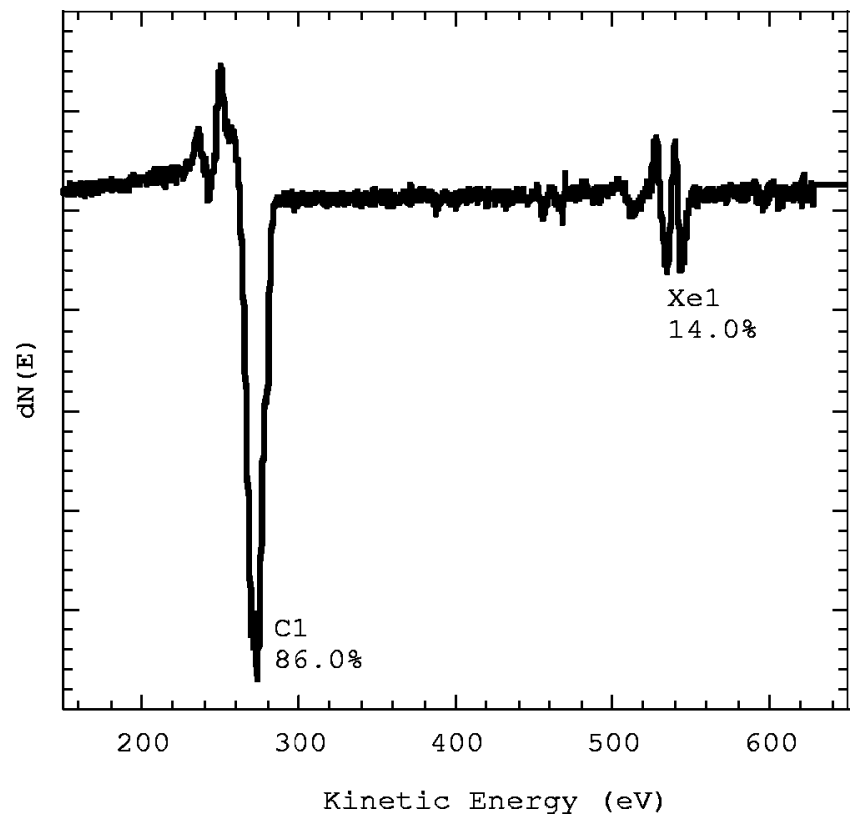

FIG. 2. AES of a graphite sample after exposure to xenon plasma bombardment reveals a significant quantity of xenon atoms trapped in the nearsurface region of the carbon lattice.

\section{EROSION MEASUREMENTS}

The sample materials used in these investigations were mounted in the biased target holder and inserted into the plasma column. The plasma parameters used in these measurements were selected to minimize the production of doubly ionized xenon, which would compromise the erosion yield results. The discharge voltage during these xenon plasma discharges was typically set to about $35 \mathrm{~V}$, resulting in an electron temperature measured in the plasma by the scanning probe of $4-5 \mathrm{eV}$. Line emission measurements of $\mathrm{Xe}^{++}$radiation was attempted, but could not be observed from the plasma under these discharge conditions. Since the second ionization potential for xenon is $33.3 \mathrm{eV},{ }^{13}$ doubly ionized xenon is energetically allowed only by a two step ionization process $\left(V_{\text {discharge }}=35 \mathrm{~V}\right)$. The small cross section for this double process and the short residence time for $\mathrm{Xe}^{+}$ in the plasma are consistent with the result of very little doubly ionized xenon ions bombarding the sample. Coupling these arguments with the sensitive nature of the spectroscopic detection technique leads us to conclude there is very little, if any, doubly ionized xenon in these PISCES plasmas.

Erosion measurements are made in two ways in PISCES. The first method is to make a standard weight-loss measurement. This technique must be corrected for any redeposition of sputtered material from ionization in the plasma back onto the sample surface, which would compromise the sputtering yield result. In the case of carbon, the amount of xenon retained in the sample must also be considered. The second measurement technique uses an absolutely calibrated optical spectrometer is used to measure the optical line emission of sputtered sample material that must pass through the plasma column. The spectroscopic measurements are based on atomic physics rate calculations from the atomic data and analysis structure (ADAS) data base. ${ }^{14}$ Each of these tech- niques requires some care, and will be discussed in detail below.

\section{A. Weight loss measurements}

The erosion yield is first measured by a standard weight loss technique. The sample is outgassed in a vacuum oven, weighted in a precision microbalance, mounted in the biased manipulator, and inserted into the plasma. The plasma discharge is started using discharge parameters that have been previously established for the desired ion flux and electron temperature. The biased sample is then bombarded with ions from the plasma for a prescribed period of time. The sample is removed, weighted, and the total erosion rate calculated from the total ion fluence and the weight loss. The sputtering yield is determined from the erosion rate corrected for any sputtered material redeposited on the sample. The fraction of eroded material redeposited on the sample is determined from the ionization mean-free path of the sputtered atoms, calculated from the plasma parameters measured by the scanning probe and verified spectroscopically. A correction to the weight loss data is made by determining the number of sputtered neutral atoms ionized in the plasma column and their subsequent transport and redeposition back onto the sample. The sputtering yield is then calculated from the net erosion rate corrected from the revised weight loss data.

The correction to the weight loss data is determined as follows. The velocity/energy of the neutral atoms sputtered from the sample surface is measured spectroscopically by examining the Doppler shift of neutral species emission line using a spectrometer view approximately normal to the sample surface. The ionization mean-free path of the sputtered atoms is then also measured spectroscopically by viewing the line averaged intensity parallel to the sample surface as a function of axial distance from the sample. This is accomplished by varying the distance between the transverse viewing location of the spectrometer and the sample surface. The $e$-folding distance of neutral molybdenum atomic line emission (normalized to the axial electron density gradient) from the plasma determines the ionization mean free path. The fit of the data reveals an ionization mean-free path for molybdenum under these plasma conditions $\left(T_{e} \approx 5 \mathrm{eV}, n_{e}\right.$ $=1.2 \times 10^{18} \mathrm{~m}^{-3}$ ) of $1.7 \mathrm{~cm}$. This agrees reasonably well with the calculated value based on the ionization rate coefficient (from $\mathrm{ADAS}^{14}$ ) of molybdenum.

The sputtered atoms can exit the plasma without being ionized, or be ionized in the plasma and either flow radially outside the sample area and be lost, or travel axially back to the sample surface and be redeposited. The number that are ionized in the plasma is determined from the ionization mean free path. Ions formed in the plasma can be lost radially before striking the sample depending on the ratio of their parallel to perpendicular velocities. The maximum axial distance away from the sample that an ion can be created and then flow back to the sample is given by

$$
z=r_{s}^{*}\left(v_{\|} / v_{\perp}\right),
$$

where $r_{s}$ is the sample radius, $v_{\|}$is the parallel flow velocity, and $v_{\perp}$ is the perpendicular ion velocity. The spectroscopic data indicates that the sputtered atom energy for Mo is about 
$2 \mathrm{eV}$, which produces $v_{\perp}=1.4 \times 10^{3} \mathrm{~m} / \mathrm{s}$. The sputtered particles with this energy can easily exit the plasma because the Larmor radius of Mo ions in the $200 \mathrm{G}$ magnetic field used during these experiments is about $7 \mathrm{~cm}$, which is much larger than the sample size. The average parallel velocity in front of the sample is assumed to be half the plasma acoustic velocity, or $v_{\perp}=\frac{1}{2} \sqrt{T_{e} / M}$. For a $5 \mathrm{eV}$ electron temperature and Mo ions, $v=1.2 \times 10^{3} \mathrm{~m} / \mathrm{s}$. For these experiments, we find $z \approx 1 \mathrm{~cm}$.

To calculate the possible fraction of sputtered atoms that can be redeposited, a cosine distribution of sputtered particles is assumed. ${ }^{15}$ This represents a worse case analysis of the number of redeposited ions because alternative distributions like those measured for $\mathrm{Xe}$ on $\mathrm{Ta}$ and $\mathrm{W}^{4}$ tend to be flatter than cosine and send the particles radially out of the plasma. The fraction of the cosine distribution that can get ionized inside the sample radius, weighted by the differential area ratio from the axis of the sample to the edge, is integrated over the surface of the sample. The maximum angle $\theta_{\max }$ at any radius inside of which an ion can hit the sample surface is given by $\theta_{\max }=\sin ^{-1}\left(r_{s}-r\right) / \lambda$. The minimum angle in the opposite direction is given by $\theta_{\min }=\sin ^{-1}\left(r_{s}\right.$ $+r) / \lambda$, where the entire distribution is ionized inside the sample radius if $r_{s}+r=\lambda$. The possible redeposition fraction is then given by

$$
F_{r}=\int_{0}^{r_{s}} \int_{0}^{2 \pi}\left[\frac{1}{2} \int_{\theta_{\min }}^{\theta_{\max }} \cos \theta d \theta\right] \frac{r d r d \phi}{\pi r_{s}^{2}} .
$$

For example, on axis at $r=0, \theta_{\max }=\sin ^{-1}\left(r_{\text {sample }} / \lambda_{\mathrm{mfp}}\right)$ $=\sin ^{-1}(1.1 / 1.7) \approx 40^{\circ}$. As $r$ is increased, the maximum angle decreases and the minimum angle increases. The integration of Eq. (2) for the $1.1 \mathrm{~cm}$ radius sample used here yields an estimated maximum redeposition fraction $F_{r}=59 \%$.

We must also correct this number by considering that only a fraction of these particles are actually ionized within an axial distance that will result in flow to the sample before the radial flow will cause them to miss the sample. This fraction can be calculated (for our Mo example) as

$$
F_{i}=\int_{0}^{z} e^{-x / \lambda} d x / \int_{0}^{\lambda} e^{-x / \lambda} d x=\frac{(1-0.56)}{(1-0.36)}=0.69 .
$$

The actual erosion rate, needed to calculate the correct sputtering yield, is obtained from the net erosion rate (i.e., the raw weight loss data) by multiplying the measured weight loss by $1 /\left(1-F_{r} F_{i}\right)$. In our case, this correction for molybdenum is a factor of 1.7. A similar correction can be derived for the titanium redeposition, resulting in $1 /\left(1-F_{r} F_{i}\right)$ correction to the weight loss data of 1.6. Due to the low mass (and therefore much faster sputtered atom velocity) of both the beryllium and carbon sputtered atoms, the ionization mean-free paths are long and the $1 /\left(1-F_{r} F_{i}\right)$ correction terms for both species are approximately equal to 1 . Note that these corrections represent the maximum redeposition amount.

Table I summarizes the sputtering yield values derived from the weight loss data including these redeposition correction terms. The sputtering yield calculated from the corrected weight loss data compares favorably with the previous
TABLE I. Summary of sputtering yields derived from weight loss data.

\begin{tabular}{cccc}
\hline \hline $\begin{array}{c}\text { Target } \\
\text { material }\end{array}$ & $\begin{array}{c}\text { Ion energy } \\
(\mathrm{eV})\end{array}$ & $\begin{array}{c}\text { Weight loss } \\
(\mu \mathrm{g})\end{array}$ & $\begin{array}{c}\text { Sputtering yield } \\
\text { (atoms/ion) }\end{array}$ \\
\hline Mo & 50 & 17510 & 0.006 \\
Mo & 50 & 420 & 0.003 \\
Mo & 100 & 11610 & 0.044 \\
Mo & 100 & 2910 & 0.035 \\
Mo & 200 & 12850 & 0.162 \\
Ti & 100 & 870 & 0.021 \\
$\mathrm{Be}$ & 100 & 420 & 0.015 \\
$\mathrm{C}$ & 50 & 20 & 0.0002 \\
$\mathrm{C}$ & 100 & 220 & 0.005 \\
$\mathrm{C}$ & 150 & 540 & 0.008 \\
\hline \hline
\end{tabular}

yield values in the literature ${ }^{2,3}$ derived from weight loss measurements at energies above $100 \mathrm{eV}$. The one discrepancy is the Mo data presented in Ref/ 4, which was an experiment actually aimed at a careful measurement of the silicon sputtering yield, and only indirectly measured the molybdenum yield. In the interpretation of the data in Ref. 4, the author emphasizes the relative values derived, but questions the obtained absolute value of the Mo sputtering yield.

A second correction to the sputtering yield derived from weight loss is the weight gained due to retention of xenon atoms within the surface. In the case of the metals, no xenon was observed in the samples after exposure [Figs. 1(a) and 1(b)], but the carbon surface contained roughly $14 \%$ xenon atoms in the surface (Fig. 2). If one assumes that at these low temperatures xenon diffusion in graphite is small, then the weight gain due to xenon trapped within the implantation depth $(\approx 1 \mathrm{~nm})$ is calculated to be less than $1 \mu \mathrm{g}$. Since the accuracy of the weight loss measurements is typically \pm 20 $\mu \mathrm{g}$, and the total weight loss is typically in the range of $100 \mathrm{~s}$ of micrograms, this correction is insignificant in this case.

A final issue that must be addressed is any surface roughening that occurs during the plasma exposure of the surfaces being exposed. Surface roughening, or cone formation, ${ }^{16}$ can lead to a reduction in the sputtering yield compared to that measured for a smooth surface. The SEM has been used to examine the exposed surfaces both before and after plasma exposure. For all the metal surfaces exposed, a smoother surface is observed after the plasma exposure, an example is shown in Fig. 3 for one of the Mo samples exposed. Poco graphite is a porous material, so no smoothing of the surface is observed due to the plasma exposure. Due to these images, no corrections are needed to account for surface morphology changes in these experiments.

\section{B. Spectroscopic measurements}

The second technique employed to measure the sputtering yield of the various materials involves an absolutely calibrated spectroscopy system. Although absolutely calibrated, the technique relies on a calculation of the excitation rate coefficient for the specific transition being monitored. The ADAS atomic data base ${ }^{14}$ includes collisional-radiative rate calculations for $\mathrm{Be}, \mathrm{C}$, and Mo line emission and ionization. The accuracy of the calculated values have been verified for 

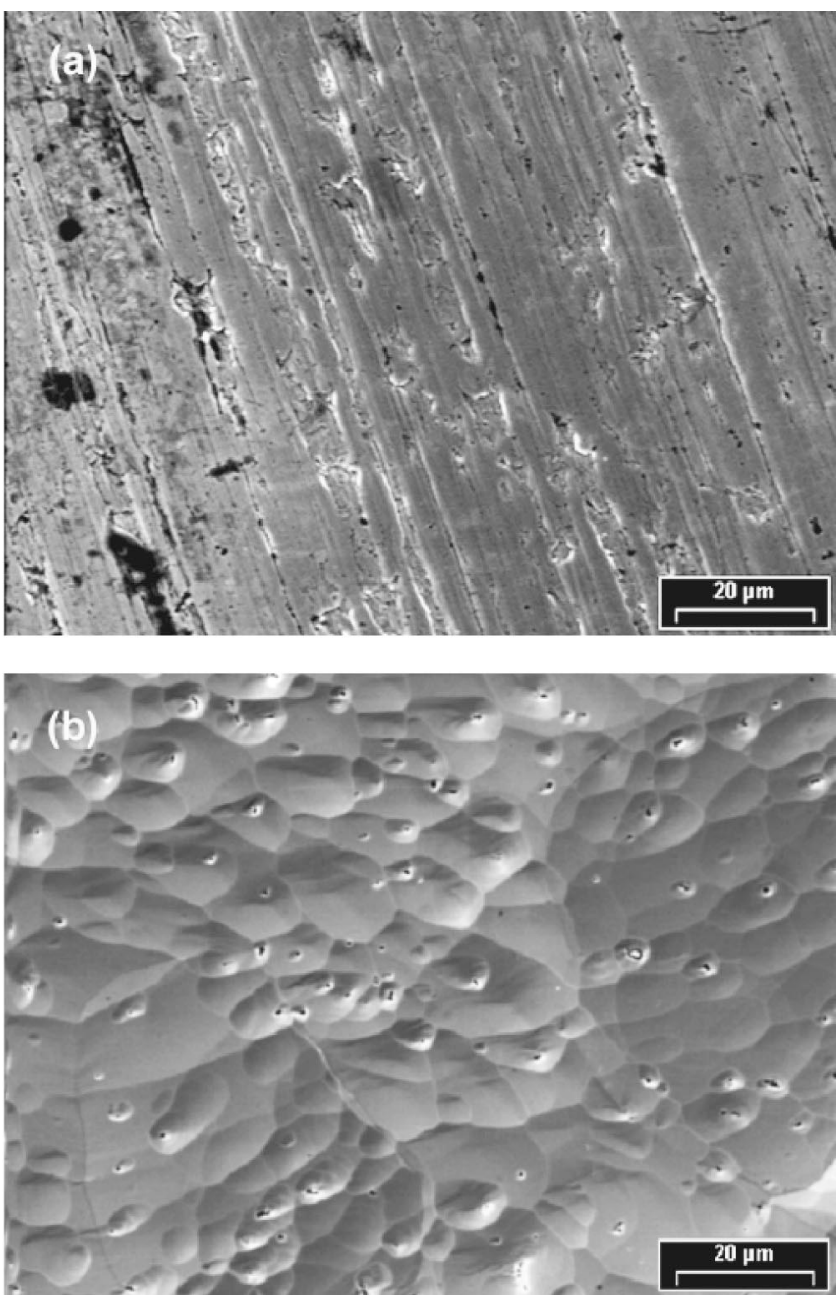

FIG. 3. SEM images of molybdenum surfaces (a) before and (b) after plasma exposure indicate that roughening of the plasma exposed surface is not occurring.

carbon ${ }^{11}$ line emission, but there has previously been no experimental verification of the calculated Be or Mo excitation rates. In addition, no atomic physics calculations have been performed for titanium. In the case of the Ti measurements, where no atomic physics calculations are available, the spectroscopic data is normalized to the weight loss data at 100 $\mathrm{eV}$. In addition, the spectroscopic technique provides a measurement of the ionization mean- free path of the sputtered target material. The weight-loss measurements discussed above provide a physical corroboration of these calculations.

The benefits of using the spectroscopic technique are threefold. First, the measurement of the sputtering yield is completed in only the few seconds integration time of the detector, as compared to the several hours of exposure needed for each weight loss data point. Second, the spectroscopic technique is much more sensitive with an extremely large dynamic range, allowing measurements down to a few tens of electron volts in ion bombarding energy. Third, the spectroscopic method is insensitive to redeposition that complicates the weight loss technique.

The spectroscopic technique is described as follows. In the case of neutral beryllium line emission, the line Be I $440.8 \mathrm{~nm}$ was monitored. Since there have been no experi-

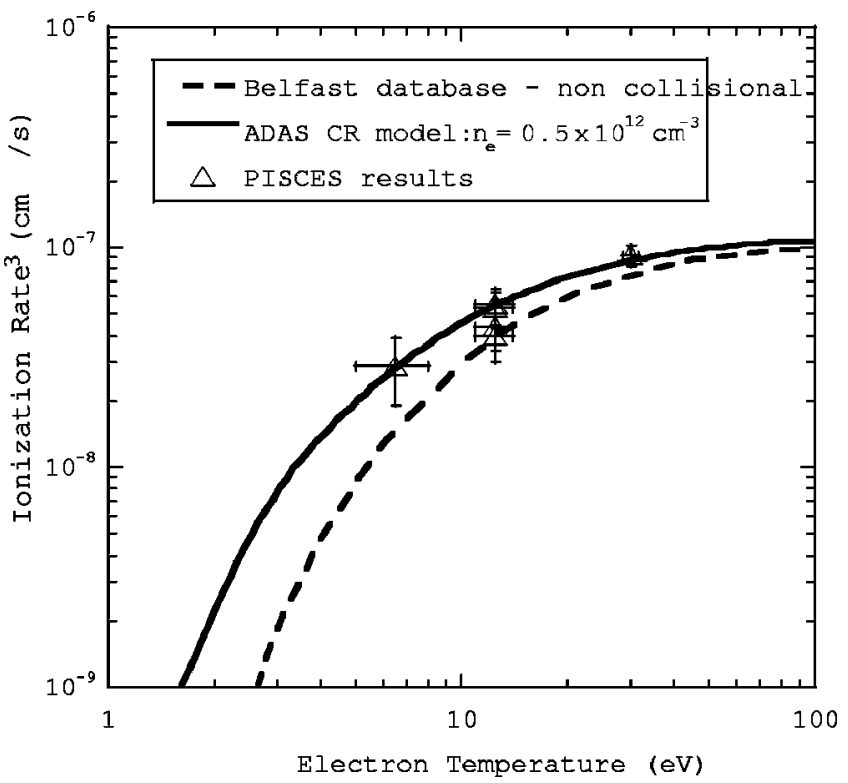

FIG. 4. Beryllium excitation rate data collected in PISCES compared to noncollisonal and CR models.

mental verification of the atomic physics calculations, the plasma parameters were varied over as wide a range as possible. To accomplish this a deuterium plasma was utilized, since the electron temperature can be varied over a wider range than when using xenon plasma. To verify the atomic rate calculations the following procedure was used. The calculated ionization rate $\langle\sigma v\rangle_{\text {ionization }}$ is defined as

$$
\langle\sigma v\rangle_{\text {ionization }}=\frac{v_{\text {eject }}}{n_{e} * \lambda_{\text {ionization }}},
$$

where $n_{e}$ is the plasma density (measured by the double Langmuir probe), $\lambda_{\text {ionization }}$ is the $e$-folding distance of the neutral Be I from the plasma (measured by collecting an axial profile spectroscopically of Be I emission and normalizing this to the axial $n_{e}$ profile), and $v_{\text {eject }}$ is the mean ejection velocity of sputtered beryllium atoms from the surface (measured using the Doppler shift spectroscopic view).

By varying the plasma conditions it is possible to control the electron temperature in the plasma column. At each plasma condition a series of measurements are made where each component of the calculated ionization rate equation is measured. The ionization rate coefficient as a function of electron temperature results. Figure 4 shows a comparison of the experimentally measured ionization rate values compared to calculated values using the ADAS collisional- radiative (CR) model $^{14}$ and the Belfast database ${ }^{17}$ that does not include CR effects. These measurements agree well with the ADAS calculations and show the importance of including CR effects in the calculation, especially for $T_{e}<20 \mathrm{eV}$ at these densities. Based on this validation in PISCES, the ADAS CR model is used for the interpretation of the spectroscopic data during the Xe plasma work.

A brief description of the spectroscopy technique to determine sputtered flux, and hence the sputtering yield, is in order. We measure the integrated brightness of sputtered neutrals leaving the surface using a view centered and $\sim$ normal 


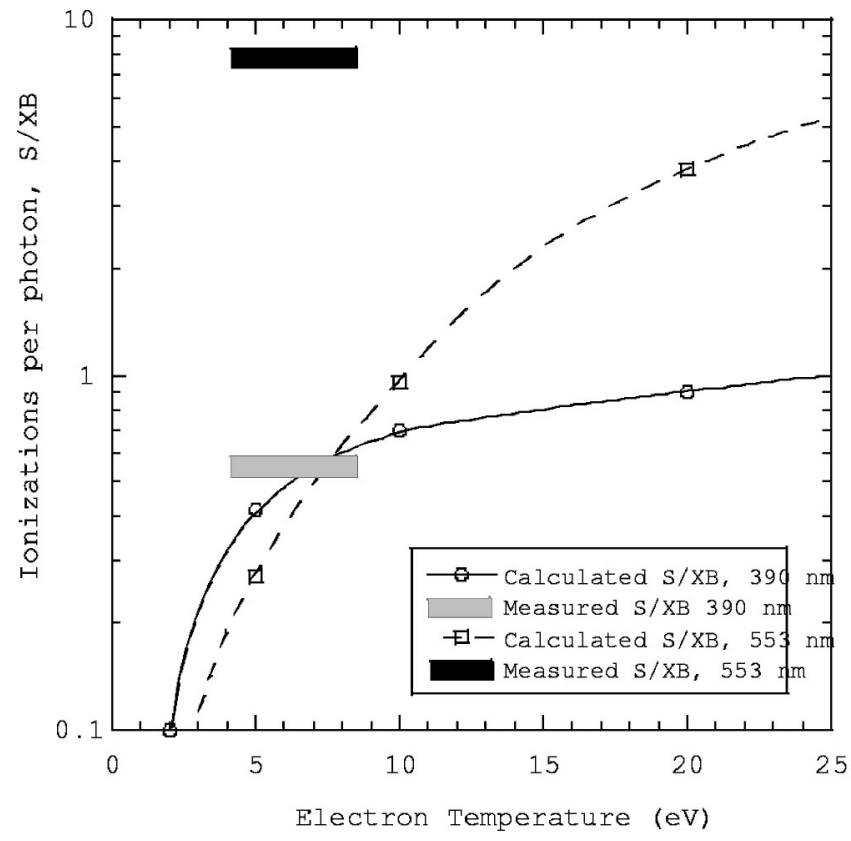

FIG. 5. ADAS calculated ionization per photon rates for Mo I. Bars indicate our experimental ionizations per photon $(S / X B)$ as benchmarked to the measured mass loss data.

to the sample surface. Inelastic collisions with the plasma electrons cause both, the excitation being measured, as well as the ionization of neutrals. If all of the sputtered neutrals are ionized within the viewing volume then the brightness (i.e., photon flux) can be converted to the neutral particle flux by simply multiplying by the calculated ratio of ionizations to excitation. This ionizations/photon ratio (traditionally called $S / X B$ ) is readily derived for a given atomic transition from the results of an atomic rate package like ADAS. One of the great strengths of this technique is that the strong exponential $T_{e}$ dependencies found in excitation rates tend to disappear since we normalize these to ionization rates that have similar $T_{e}$ dependencies.

In the case of molybdenum, line emissions at 553.3 and $390.3 \mathrm{~nm}$ were monitored. The most desirable transition to use for absolute spectroscopy is a transition with little electron temperature dependence. This is true of the $390 \mathrm{~nm}$ transition which is directly excited from and decays into the ground state (making the atomic physics rate calculations more reliable) with an energy threshold of only $\sim 2.3 \mathrm{eV}$. This makes the ionizations per photon approximately constant for $T_{e}>4 \mathrm{eV}$ (see Fig. 5). An undesirable strong electron temperature dependence for $553 \mathrm{~nm}$ transition is expected from the ADAS calculations (also it is not a groundstate transition).

Unfortunately, the $390 \mathrm{~nm}$ transition is in close proximity to the PISCES-B window spectral cutoff, making it unsuitable except for high-yield or high-bias conditions with a high flux of sputtered molybdenum atoms. Therefore, we have used the $390 \mathrm{~nm}$ transition to determine an absolute sputtering yield of $\mathrm{Xe}$ on Mo at a single bias condition (100 $\mathrm{V}$ incident ion energy), resulting in a yield of $3 \% \pm 1 \%$ using the calculated ADAS rates. This yield value is approximately $50 \%$ lower than the weight loss measurements presented in
TABLE II. Spectroscopic yield data (Ti data are normalized to weight loss @ $100 \mathrm{eV}$ ).

\begin{tabular}{|c|c|c|c|c|c|}
\hline $\begin{array}{c}\text { Ion } \\
\text { energy } \\
(\mathrm{eV})\end{array}$ & $\begin{array}{l}Y(\mathrm{Mo}) \\
\text { atoms }\end{array}$ & $\begin{array}{l}Y(\mathrm{Ti}) \\
\text { atoms }\end{array}$ & $\begin{array}{l}Y(\mathrm{Be}) \\
\text { atoms }\end{array}$ & $\begin{array}{l}Y(\mathrm{C}) \\
\text { atoms }\end{array}$ & $\begin{array}{c}Y\left(\mathrm{C}_{2}\right) \\
\text { molecules }\end{array}$ \\
\hline 200 & 0.082 & $\cdots$ & 0.025 & $\cdots$ & $\cdots$ \\
\hline 175 & 0.072 & $\ldots$ & 0.023 & $\ldots$ & \\
\hline 150 & 0.059 & 0.045 & 0.017 & $4.6 \times 10^{-5}$ & 0.00068 \\
\hline 125 & 0.045 & $\ldots$ & 0.012 & $2.4 \times 10^{-5}$ & 0.00040 \\
\hline 100 & 0.03 & 0.021 & 0.008 & $2.2 \times 10^{-5}$ & 0.00030 \\
\hline 90 & 0.023 & $\cdots$ & $\cdots$ & $\cdots$ & 0.00026 \\
\hline 80 & 0.017 & 0.014 & $\cdots$ & $1.0 \times 10^{-5}$ & 0.00022 \\
\hline 80 & 0.018 & $\cdots$ & $\cdots$ & $\cdots$ & $\cdots$ \\
\hline 75 & $\cdots$ & $\cdots$ & 0.0044 & $\cdots$ & $\cdots$ \\
\hline 70 & 0.011 & $\cdots$ & $\cdots$ & $\cdots$ & 0.00020 \\
\hline 60 & 0.0069 & 0.0052 & $\ldots$ & $3.0 \times 10^{-6}$ & 0.00018 \\
\hline 60 & 0.0087 & $\cdots$ & $\cdots$ & $\cdots$ & $\cdots$ \\
\hline 50 & 0.0036 & 0.0025 & 0.00084 & $\cdots$ & 0.00016 \\
\hline 50 & 0.0051 & $\cdots$ & $\cdots$ & $\cdots$ & $\cdots$ \\
\hline 45 & 0.0022 & $\cdots$ & $\cdots$ & $\cdots$ & $\cdots$ \\
\hline 40 & 0.0021 & 0.00079 & $\cdots$ & $\cdots$ & 0.00015 \\
\hline 40 & 0.0013 & $\ldots$ & $\ldots$ & $\cdots$ & $\cdots$ \\
\hline 35 & 0.00068 & $\cdots$ & $9.0 \times 10^{-5}$ & $\ldots$ & $\ldots$ \\
\hline 30 & 0.00051 & 0.00021 & $\ldots$ & $\cdots$ & 0.00014 \\
\hline 30 & 0.00032 & $\cdots$ & $\cdots$ & $\cdots$ & $\cdots$ \\
\hline 30 & 0.00030 & $\cdots$ & $\cdots$ & $\cdots$ & $\cdots$ \\
\hline 25 & 0.00016 & $\ldots$ & $3.0 \times 10^{-6}$ & $\ldots$ & $\ldots$ \\
\hline 25 & $9.2 \times 10^{-5}$ & $\ldots$ & $\ldots$ & $\ldots$ & $\ldots$ \\
\hline 20 & $1.7 \times 10^{-5}$ & $2.5 \times 10^{-5}$ & $\cdots$ & & 0.00011 \\
\hline 20 & $\cdots$ & $1.7 \times 10^{-5}$ & $\cdots$ & $\cdots$ & $\cdots$ \\
\hline 15 & $2.5 \times 10^{-7}$ & $2.5 \times 10^{-7}$ & $\ldots$ & $\ldots$ & $\ldots$ \\
\hline 10 & $\ldots$ & $2.5 \times 10^{-7}$ & $\cdots$ & $\ldots$ & $7.6 \times 10^{-5}$ \\
\hline
\end{tabular}

Table I, but this discrepancy is likely within the usual $25 \%-$ $50 \%$ uncertainty in the atomic rate calculations. The large experimental uncertainty primarily results from the difficult absolute calibration near the spectral cutoff. Attempting a similar measurement using the $553 \mathrm{~nm}$ line results in a sputtering yield that is a factor of about 30 too low. That is to say, that the experimentally measured ratio of the Mo I $553 \mathrm{~nm}$ brightness to the Mo I $390 \mathrm{~nm}$ brightness is 30 times smaller than predicted from ADAS. Figure 5 compares the ADAS calculated ionizations per photon $(S / X B$, calculated at a plasma density of $1 \times 10^{13} \mathrm{~cm}^{-3}$ ) with the experimentally measured values when we assume the sputtering yield derived from the weight loss technique. Fortunately, for fixed plasma conditions, any Mo I line brightness is linearly dependent on Mo erosion rate, so the $553 \mathrm{~nm}$ transition is used (normalized to the brightness of the $390 \mathrm{~nm}$ line) for obtaining the sputtering yield dependence on incident ion energy. This result seems to point at some deficiency in the CR model of ADAS at low $T_{e}$ for the $553 \mathrm{~nm}$ transition.

For the remaining materials tested, appropriate spectral lines were used. For titanium, the spectroscopic measurement used the neutral titanium atom line emission at 499.1 nm. And finally, neutral carbon atom line emission at 833.5 and $940.5 \mathrm{~nm}$ were used. In addition, molecular band emission from $\mathrm{C}_{2}$ molecules (the Swan band near $516.5 \mathrm{~nm}$ ) was measured (for reasons explained in the next sections). Values of the sputtering yield obtained spectroscopically are presented in Table II. 


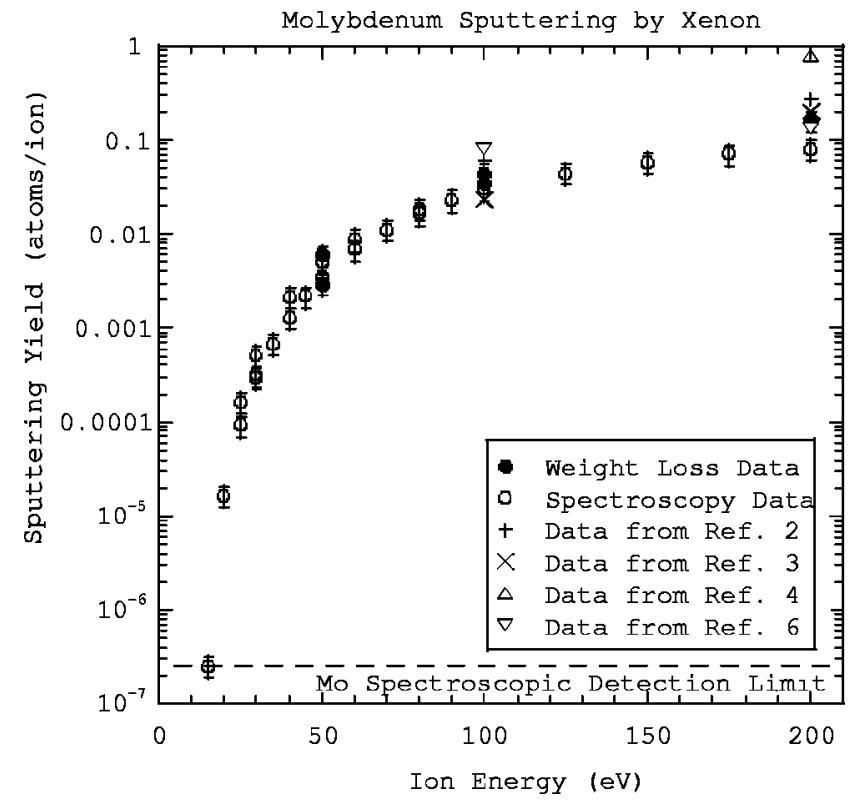

FIG. 6. Comparison of present molybdenum sputtering yield data listed in Tables I and II with earlier molybdenum erosion measurements.

The interpretation of the measurements for the metals investigated is straightforward. Whether the data is based upon the absolute spectroscopic technique based on atomic physics rate calculations (in the case of Mo and $\mathrm{Be}$ ) or simply spectroscopic signals scaled to weight loss data (for Ti), this data extends the database to lower energies than previously available.

\section{SPUTTERING YIELD RESULTS}

\section{A. Metals erosion measurements}

A comparison of the sputtering yield measured here for molybdenum with the results of Refs. 2 and 3 is shown in Fig. 6. The PISCES data are observed to lie between the data points from the literature at energies of $100 \mathrm{eV}$ and above, and extend the yield measurements down nearly 6 orders of magnitude to the detection limit of the spectrometer. Based on these results, the threshold for xenon sputtering of molybdenum is on the order of $15 \mathrm{eV}$.

The sputtering yield data for Ti and Be measured in PISCES are shown in Fig. 7. Since titanium and beryllium have a much larger mass difference compared to the molyxenon combination, the yields measured for these metals are reduced accordingly. At $100 \mathrm{eV}$, titanium sputters about 1.9 times slower than molybdenum, and beryllium sputters about 2.6 times slower.

\section{B. Carbon erosion measurements}

The sputtering yield results for xenon ion bombardment of Poco graphite are compared to the molybdenum sputtering yield in Fig. 8. Based on the weight loss measurements, carbon is found to erode at a rate about 8 times slower than molybdenum at $100 \mathrm{eV}$ and over a factor of 20 times slower than molybdenum at $50 \mathrm{eV}$. These results are consistent with results in the literature for $100 \mathrm{eV}$ and above. However, the spectroscopic techniques, which observe the individual car-

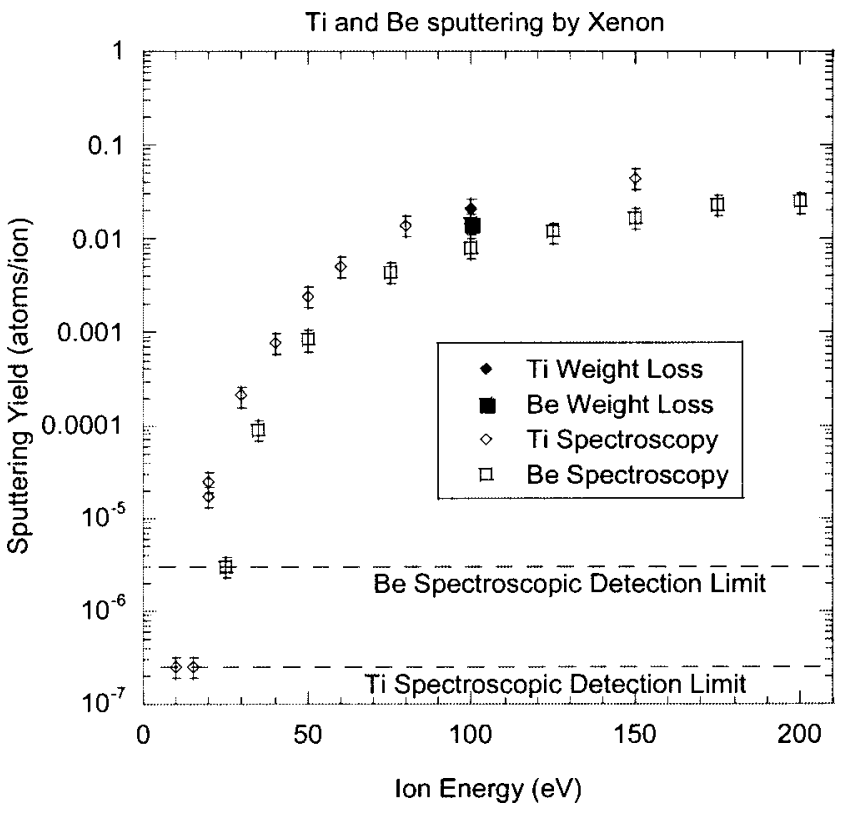

FIG. 7. Graphical representation of the Ti and Be sputtering yield data listed in Tables I and II.

bon atom and the sputtered carbon dimer $\left(\mathrm{C}_{2}\right)$ lines, shows erosion yields significantly lower than that found with the weight loss technique for the total sample.

The data obtained during the spectroscopic measurements on carbon samples requires additional interpretation. First, recall that the ADAS calculations for carbon-atom lines have already been benchmarked for this transition ${ }^{11}$ and

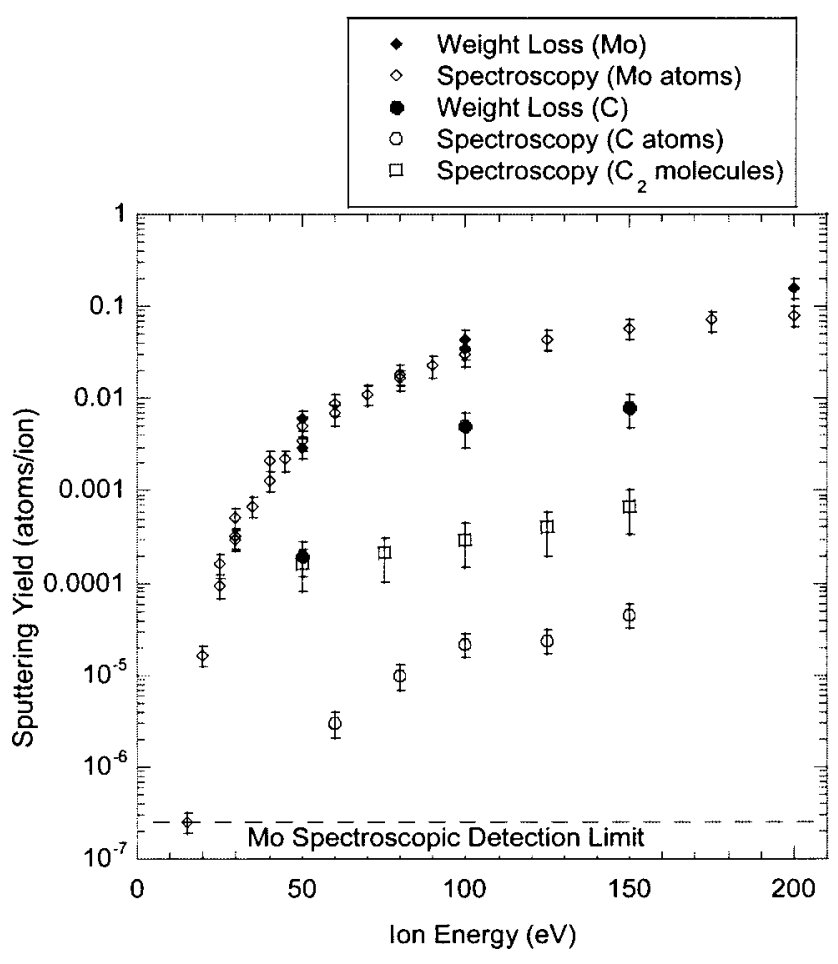

FIG. 8. Comparison of sputtering yield data from molybdenum and carbon. The importance of dimer sputtering is seen to be larger than atomic carbon sputtering during xenon ion bombardment. 
have been used extensively in fusion research. This means that the amount of carbon sputtered as atoms is well characterized and is significantly smaller than is required to match the weight loss technique. One possible explanation could be the influence of sputtering of clusters of carbon atoms from the surface during xenon ion bombardment. It may be reasonable to hypothesize that because of the large mass difference, and therefore poor energy transfer, between the incident $\mathrm{Xe}$ ions and the carbon atoms of the target, that preferential sputtering of higher mass clusters of carbon atoms could occur. Alternatively, it could be possible that stresses to the graphite lattice due to the inclusion of large numbers of xenon atoms may increase the likelihood of sputtering clusters of carbon atoms from the surface during bombardment.

The efflux of carbon dimers (i.e., $\mathrm{C}_{2}$ ) is determined from spectroscopic measurement of the $\mathrm{C}_{2}$ molecular resonance band (Swan band, $516.5 \mathrm{~nm}$ ) and using the calculated photons per molecule ${ }^{18}$ of $\sim 2$ (for $T_{e} \sim 5 \mathrm{eV}$ ). The results indicate that $\mathrm{C}_{2}$ clusters are being sputtered with a yield approximately ten times larger than that of individual carbon atoms. Typically, the sputtering yield for clusters exhibits a power law decay with the increasing number of atoms in the cluster. ${ }^{19}$ While significantly larger than the sputtering yield of carbon atoms, $\mathrm{C}_{2}$ sputtering is still too small to account for the entire weight loss measurement at incident ion energies above $50 \mathrm{eV}$. It is possible that other higher order carbon clusters also contribute to the erosion of the sample, but we are not aware of a plasma-based technique to measure larger clusters. Previous measurements of carbon cluster sputtering showed similar amounts of $\mathrm{C}_{2}$ and $\mathrm{C}_{3}$ clusters emitted during argon ion bombardment. ${ }^{20}$

During any investigation of erosion of carbon one must also be careful to evaluate the impact of residual amounts of oxygen impurity ions striking the surface along with the primary plasma species, in this case xenon. The chemical erosion yield of high-energy oxygen ions on carbon, in this temperature regime, is approximately 0.4 due to the formation of volatile CO molecules. ${ }^{21}$ This would lead to an estimated weight loss yield, due to chemical erosion, of 0.0004 due to the base pressure in PISCES-B. The weight loss expected from chemical erosion by residual oxygen is within the error of the weight loss measurement at $50 \mathrm{eV}$ for carbon, and clearly much less than the carbon yield measured by weight loss at 100 and $150 \mathrm{eV}$.

\section{SUMMARY}

A plasma based spectroscopic technique has been used to extend the low-energy range of sputtering yield measurements for ion thruster relevant materials and conditions. Sputtering yields as low as $2.5 \times 10^{-7}$ atoms/ion for $15 \mathrm{eV}$ xenon ion bombardment of molybdenum have been mea- sured. The spectroscopic technique compares well with weight loss measurements made after the conclusion of the plasma exposure. The yields derived from the weight loss measurements performed here fall within the scatter of previous erosion yield measurements in the literature at energies of $100 \mathrm{eV}$ and higher.

Similar results are presented for xenon plasma bombardment of titanium, beryllium, and for carbon erosion based on weight loss measurements. Only the spectroscopic data for carbon showed unexpected results. Chemical erosion of the graphite samples from residual water vapor in the vacuum chamber is insufficient to explain the weight loss measurements of the carbon samples. Erosion of carbon clusters from the sample is a possible explanation for the observed weight loss. $\mathrm{C}_{2}$ clusters are measured to contribute a factor of 10 more to the weight loss than atomic carbon sputtering. Higher order cluster ejection may also contribute to the weight loss, but confirmation of this hypothesis will require further dedicated measurements in ion beam facilities.

\section{ACKNOWLEDGMENTS}

The authors gratefully acknowledge the technical support provided by members of the PISCES Laboratory staff, without whom none of the research would have been possible. This research was supported by Boeing Electronic Dynamic Devices, Inc.

${ }^{1}$ J. J. Blandino, D. G. Goodwin, and C. E. Garner, Diamond Relat. Mater. 9, 1992 (2000).

${ }^{2}$ D. Rosenberg and G. K. Wehner, J. Appl. Phys. 33, 1842 (1962).

${ }^{3}$ C. H. Weijsenfeld, A. Hoogendoorn, and M. Koedam, Physica (Amsterdam) 27, 763 (1961).

${ }^{4}$ P. C. Zalm, J. Appl. Phys. 54, 2660 (1983).

${ }^{5}$ M. A. Mantenieks, P. K. Ray, and S. V. Shutthanandan, Proceedings of 27th International Electric Propulsion Conference, Pasadena, CA, Oct. 2001, pp. 15-19.

${ }^{6}$ O. B. Duchemin and J. E. Polk, AIAA Pap. , 99-2858 (1999).

${ }^{7}$ D. M. Goebel, G. Campbell, and R. W. Conn, J. Nucl. Mater. 121, 277 (1984).

${ }^{8}$ D. G. Whtye et al., Nucl. Fusion 41, 47 (2001).

${ }^{9}$ R. Doerner and C. Mays, Fusion Eng. Des. 37, 325 (1997).

${ }^{10}$ S. C. Luckhardt et al., J. Nucl. Mater. 266-269, 1285 (1999).

${ }^{11}$ D. G. Whyte, R. Seraydarian, and R. P. Doerner, J. Vac. Sci. Technol. A 17, 2713 (1999)

${ }^{12}$ F. C. Sze, R. P. Doerner, and S. Luckhardt, J. Nucl. Mater. 264, 89 (1999).

${ }^{13}$ C. Kittel, Introduction to Solid State Physics (Wiley, New York, 1976).

${ }^{14}$ H. P. Summers, Atomic Data and Analysis Structure-User Manual, Rep. JET-IR(94) (JET Joint Undertaking, Abingdon, 1994).

${ }^{15}$ Sputtering by Particle Bombardment I, edited by R. Behrisch, Topics in Applied Physics, Vol. 47 (Springer, Berlin, 1981).

${ }^{16}$ Sputtering by Particle Bombardment II, edited by R. Behrisch, Topics in Appl. Physics, Vol. 52 (Springer, Berlin, 1983).

${ }^{17}$ M. A. Lennon et al., J. Phys. Chem. Ref. Data 17, 1285 (1988).

${ }^{18}$ R. C. Isler et al., Phys. Plasmas 8, 4470 (2001).

${ }^{19}$ A. Wucher, M. Wahl, and H. Oechsner, Nucl. Instrum. Methods Phys. Res. B 82, 337 (1993).

${ }^{20}$ E. Vietzke et al., J. Nucl. Mater. 241-243, 810 (1997).

${ }^{21}$ E. Vietzke et al., J. Nucl. Mater. 145-147, 425 (1987). 